\title{
ANGIOGRAPHIC DIAGNOSIS OF VERTEBRAL ARTERY THROMBOSIS*
}

\author{
BY \\ A. VAN DER ZWAN
}

\section{From the Department of Neurosurgery, University Hospital, Groningen, Holland}

The diagnosis of vascular lesions of the brain may be very difficult : clinicians frequently see cases in which the mode of onset and the clinical features suggest that a haemorrhage or thrombosis has occurred, and yet the later developments show that the symptoms have been due to a tumour. Conversely, there are cases in which the insidious onset and apparent progression of symptoms might reasonably be ascribed to a tumour, but further investigation reveals instead a vascular lesion. With increasing interest in the active treatment of some of these lesions, differential diagnosis becomes even more important. In the case of lesions of the cerebral hemisphere, carotid angiography has proved to be a valuable expedient, and it is usually possible to say on the basis of this comparatively simple procedure whether we are dealing with neoplasm, haemorrhage, or clot. For lesions below the tentorium, however, angiography of the vertebral system is less reliable: the technique is more difficult, and in cases of tumour or vascular occlusion, it might be dangerous.

There are, however, some circumstances under which carotid angiography demonstrates the vertebral system of arteries, and this report is concerned with two such cases, in both of which the basilar artery filled via the posterior communicating arteries owing to thrombosis of the lower parts of the vertebral system. $\dagger$

\section{Case Reports}

Case 1.-The first patient was a man of 56 years with a very scanty history. His wife said that his mentality and character had been deteriorating for about 12 months. He was forgetful, irritable, and uninhibited and his manners became progressively more asocial. Apart from a pathological appetite, he had no physical symptoms until three months before admission when he began to have occasional attacks of giddiness and vomiting. On the day before admission to hospital he became very drowsy, and he was sent into hospital under suspicion of

* Based on a paper read to the Society of British Neurological Surgeons in May, 1953.

† Since this paper was prepared, three further cases have been seen. having a frontal lobe tumour. On admission he was drowsy and confused and had persistent hiccough. No focal neurological abnormalities were detected and there was no objective evidence of increased intracranial pressure. The blood pressure was $180 / 120 \mathrm{~mm}$. $\mathrm{Hg}$ and the blood urea and blood sugar levels were normal. A carotid angiogram (see below) showed no evidence of tumour, and as the diagnosis was still uncertain a ventriculogram was done. This likewise showed no evidence of a tumour, but there were curious collections of air in the region of the incisura tentorii which persisted despite alteration in posture of the head. It was thought that this appearance might be due to subarachnoid pouch and the region was accordingly explored through a right occipital osteoplastic flap. No definite abnormalities were found and the patient died on the following day.

At necropsy, the diagnosis was immediately apparent : there was thrombosis of both vertebral arteries, the left posterior inferior cerebellar artery, and a recent clot in the basilar artery. All these vessels were markedly atheromatous, although the carotid system appeared to be normal. There was acute ischaemic softening of both cerebellar lobes and of the left side of the medulla ; the cerebral hemispheres, basal ganglia, and brain-stem were all normal.

This case showed little of the common clinical features of vertebral or basilar occlusion but the history and examination were necessarily limited by the rapid terminal collapse. When the arteriogram was reviewed, I was struck by the fact that the upper part of the basilar artery was well filled from the carotid injection, and it seemed likely that this might be of diagnostic value in future cases.

Case 2.-The second patient was a woman, 52 years old, who had been known to have high blood pressure for five years. Four years before admission, there was an episode of acute pain in the neck which lasted for a week and then cleared up completely. For several weeks before she came into hospital she complained of recurrent numbness of the right little finger, each bout lasting two or three days. Two days before admission, she had an attack of giddiness while riding a bicycle. On the day of admission she woke up with acute vertigo and vomiting, and it was these symptoms which led to her being sent to hospital. 
On admission, she was somewhat cyanosed and short of breath, and she looked ill. She was very excited, talking loudly in an uninhibited manner. The head was kept turned to the right but the neck was not stiff. The positive neurological abnormalities amounted to paralysis of conjugate lateral movements of the eyeballs with rapid rotatory nystagmus on looking to the right and upwards ; impaired sensation in the left trigeminal zone; a left peripheral facial palsy ; a relative increase in the tendon reflexes in the right limbs, and slight ataxy of the left limbs. There was no evidence of increased intracranial pressure. The blood pressure was $180 / 120 \mathrm{~mm}$. $\mathrm{Hg}$.

These clinical features pointed to thrombosis of the left posterior inferior cerebellar artery with probable partial occlusion of the basilar artery. The patient was treated with heparin and nicotinic acid and rapidly improved. She was discharged from hospital at the end of two months with no neurological abnormalities except slight unsteadiness of gait.

Nine months later she was readmitted in a very similar attack characterized by excitement, dysarthria, rapid and irregular respiration, nystagmus, spastic weakness of the right limbs, and ataxy of the left limbs. On this occasion a carotid angiogram was done and in addition to the normal carotid circulation, the upper part of the basilar and the superior cerebellar arteries were visualized as in the first case (Fig. 1).

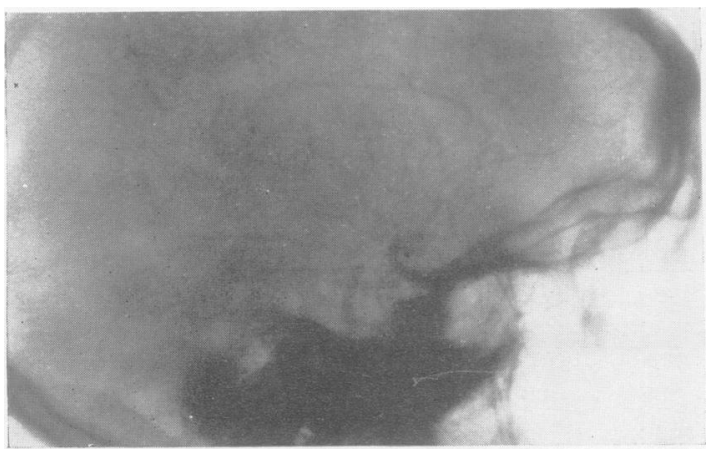

Fig. 1.-Carotid arteriogram of Case 2.

Again there was rapid improvement with medical treatment, and when seen one and a half years later she appeared to be well and had no residual neurological deficit except slight unsteadiness of gait. It was noted, however, that if her head was bent backwards, she complained of giddiness and nystagmus developed, features described by Biemond (1951) as being characteristic of obstructed circulation in the vertebral arterial system.

\section{Comment}

Filling of the basilar artery with contrast medium injected into the common carotid artery occurred in these two cases by way of the posterior communicating artery. If the vertebral or proximal part of the basilar artery is occluded, the contrast medium in the carotid system has less pressure to meet and the possibility of such reflex filling would be enhanced.
In a series of 500 carotid angiograms I have seen filling of the basilar artery in only two other cases. The first was in a patient with thrombosis of the internal carotid artery. As the external carotid circulation could not take all the contrast medium, some of it flowed back in the common carotid artery and reached the basilar by way of the vertebral artery. Moniz (1940) and Krayenbühl and Richter (1952) have described similar cases. The second case was that of a child with bilateral subdural haematoma. A right carotid angiogram filled the whole intracranial arterial tree (Fig. 2), i.e., the vessels of

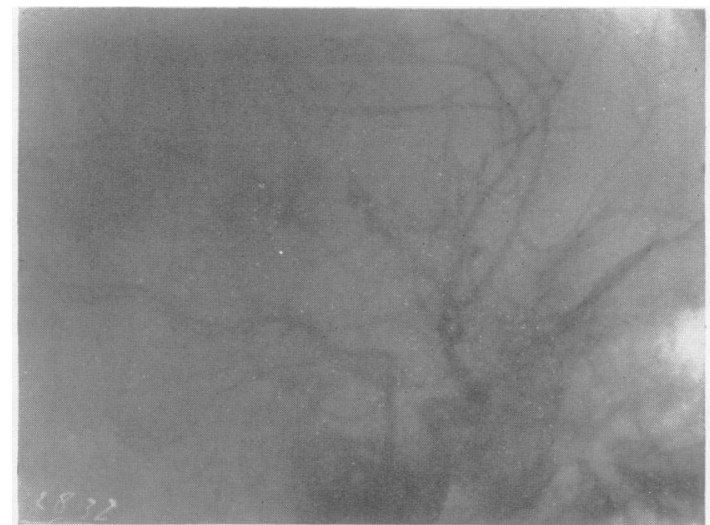

FIG. 2.-Carotid arteriogram in a case of bilateral subdural haematoma, showing good filling of the basilar artery and its branches.

the right and left cerebral hemispheres, as well as the basilar and vertebral arteries. Moniz has also described this finding in some cases of high intracranial pressure, and certainly the pressure was very high in our case. But as increased intracranial pressure could be excluded in the two cases reported above, it seems likely that when the basilar artery is visualized by carotid angiography some obstruction in the vertebral artery should be considered.

In many cases of thrombosis of the vertebral, posterior inferior cerebellar and basilar arteries, the clinical features are sufficient for the diagnosis. But in doubtful or obscure cases it is suggested that carotid angiography may prove helpful not only by excluding a cerebral tumour but also by demonstrating filling of the basilar artery.

\section{Summary}

Two cases are reported of thrombosis of the vertebral artery system in which carotid angiography filled the basilar artery via the posterior communicating artery.

\section{REFERENCES}

Biemond, A. (1951). Brain, 74, 300.

Krayentühl, H., and Richter, H. R. (1952), Die Zerebrale Angiographie. Georg Thieme, Stuttgart.

Moniz, E. (1940). Die Cerebrale Arteriographic und Phlebographie. Handbuch der Neurologie. ed. Bumke, O. and Foerster, O., Ergänzungsserie II. Springer, Berlin. 